\title{
Enhanced expression of natriuretic peptide receptor $A$ and $B$ in neutrophils of culprit lesions in patients with acute myocardial infarction
}

\author{
MASASHI NAKAGAWA ${ }^{1}$, TAKAHIKO NARUKO ${ }^{2}$, KENICHI SUGIOKA ${ }^{1}$, \\ CHIZUKO KITABAYASHI ${ }^{3}$, NOBUYUKI SHIRAI ${ }^{1}$, MASAHIKO TAKAGI $^{1}$, \\ MINORU YOSHIYAMA ${ }^{1}$, MASAHIKO OHSAWA ${ }^{4}$ and MAKIKO UEDA ${ }^{5}$
}

\footnotetext{
${ }^{1}$ Department of Cardiovascular Medicine, Osaka City University Graduate School of Medicine, Osaka 545-8585;

${ }^{2}$ Department of Cardiology, ${ }^{3}$ Division of Nephrology and Hypertension, Osaka City General Hospital, Osaka 534-0021;

${ }^{4}$ Department of Pathology, Osaka City University Graduate School of Medicine, Osaka 545-8585;

${ }^{5}$ Department of Medical Technology, Morinomiya University of Medical Sciences, Osaka 559-8611, Japan
}

Received April 15, 2016; Accepted May 9, 2017

DOI: $10.3892 / \mathrm{mmr} .2017 .7034$

\begin{abstract}
Natriuretic peptides and their specific receptors have been suggested to have regulatory effects on smooth muscle cell (SMC) growth and inflammatory cell reactions. However, the roles of natriuretic peptide receptor A (NPR-A) and B (NPR-B) in unstable plaques remain to be studied in detail. Frozen sections from 82 coronary artery segments were used. These segments were obtained at autopsy from 13 patients with acute myocardial infarction (AMI; 7 ruptured and 6 eroded plaques) and from 30 patients with non-cardiovascular diseases. Antibodies against SMCs, endothelial cells, macrophages, neutrophils, NPR-A, NPR-B and neutral endopeptidase (NEP) were used. Neutrophil infiltration was identified in all lesions with plaque rupture or erosion. Double immunostaining identified that the majority of NPR-A- or NPR-B-positive cells were neutrophils in ruptured and eroded plaques. Using morphometric analysis, no significant difference was observed in the percentage of NPR-A- and NPR-B-positive cells between ruptured and eroded plaques, while the number of NEP-positive neutrophils in ruptured plaques was significantly higher compared with eroded
\end{abstract}

Correspondence to: Dr Masashi Nakagawa, Department of Cardiovascular Medicine, Osaka City University Graduate School of Medicine, 1-4-3 Asahi-machi, Abeno-ku, Osaka 545-8585, Japan E-mail: m-nakagawa@med.osaka-cu.ac.jp

Abbreviations: SMC, smooth muscle cell; AMI, acute myocardial infarction; NPR-A, natriuretic peptide receptor-A; NPR-B, natriuretic peptide receptor-B; NEP, neutral endopeptidase; NP, natriuretic peptides; CNP, C-type natriuretic peptide; PCI, percutaneous coronary intervention; cGMP, cyclic guanosine monophosphate

Key words: natriuretic peptide, smooth muscle cells, inflammation, atherosclerosis, plaque instability plaques $(\mathrm{P}<0.0001)$. These results of the distinct presence of NPR-A- and NPR-B-positive cells in unstable plaques underlying AMI suggested that natriuretic peptides serve a role in regulating plaque instability in humans.

\section{Introduction}

Atherosclerotic plaque injuries and intraplaque inflammation are widely considered to serve a crucial role in the development of acute coronary syndromes (1-3). It has been demonstrated that vulnerable plaques of human coronary arteries contain abundant macrophages and T lymphocytes (3). The authors of the present study previously demonstrated that distinct accumulation of neutrophils additionally occurs in culprit lesions of acute coronary syndromes $(4,5)$. Activated neutrophils can release enzymes, including elastase and myeloperoxidase. Neutral endopeptidase 24.11 (NEP), a membrane protein that regulates inflammatory reactions, can also be identified in neutrophils. NEP can hydrolyze a large number of peptides, including the natriuretic peptide (NP) family (6). It has already been reported (4) that neutrophils were positive for NEP in ruptured plaques, while in eroded plaques the majority of neutrophils lacked NEP positivity.

Previous epidemiological studies have identified that the level of NPs in the peripheral blood correlates positively with the risk of coronary atherosclerosis and acute myocardial infarction (AMI) $(7,8)$. Two types of NP receptors (NPRs), the biologically active receptor and the clearance receptor, have been reported and the biologically active receptors are further classified into two subtypes, NPR-A and NPR-B $(9,10)$. A previous study of human coronary atherosclerotic lesions demonstrated that smooth muscle cells (SMCs) in early atherosclerotic hypercellular lesions were positive for C-type NP (CNP), whereas SMCs in advanced lesions were negative for CNP (11). Furthermore, it has been reported by examination of autopsy specimens, in addition to atherectomy specimens, that the expression of NPRs was detected in neointimal SMCs at the site of percutaneous coronary intervention (PCI) (12). 
Experimental studies have demonstrated that neutrophils express the biologically active receptors of NPRs, which limit neutrophil activation via the generation of intracellular cyclic guanosine monophosphate (cGMP) (13). Although the effects of NPs on neutrophils remain unclear, previous experimental studies reported the priming of superoxide anions in neutrophils by atrial NP (ANP) and by brain NP $(14,15)$. In addition, Izumi et al (16) demonstrated the advantageous result of the blocking of NPR-A in models of ischemia/reperfusion in mice.

Therefore, identification of the cellular localization of NPR-A and -B is important for clarifying the pathophysiological role of these receptors in plaque instability. However, the localization of NPR-A and -B in the coronary culprit lesions of AMI remains to be reported. The present study provides information on the immunohistochemical localization that was used to define the pattern of NPR-A and -B expression in ruptured and eroded plaques from patients with AMI.

\section{Materials and methods}

Coronary tissue specimens. A total of 82 coronary artery segments were collected at autopsy from 43 patients (13 segments from 13 patients with AMI, 69 segments from 30 patients with non-cardiovascular diseases). Each segment was obtained within $3 \mathrm{~h}$ following mortality. The age range of these patients was between 11 and 74 years (non-cardiovascular disease $54 \pm 19 \mathrm{yr}$; AMI 50 \pm 17 years, mean \pm standard deviation). These two study groups were composed predominantly of males [non-cardiovascular disease (males, 25; females 5); AMI 77\% (males, 10; females, 3)]. In the 69 segments obtained from the patients with non-cardiovascular diseases, 20 segments contained normal coronary arteries with diffuse intimal thickening (American Heart Association classification type I) $(17,18)$ and 49 segments contained atherosclerotic lesions. These atherosclerotic lesions were classified histologically according to the previously described system (11) either as early atherosclerotic lesions with hypercellularity $(n=12)$ or as advanced atherosclerotic lesions $(n=37)$. The advanced atherosclerotic lesions were further classified into two types; fibrolipid (type Va; $n=22$ ) or fibrous (type Vc; $n=15$ ). The definition of these various types of atherosclerotic lesions has been described previously $(4,11)$. The current study was approved by the Osaka City General Hospital Ethical Committee (approval number 606; Osaka, Japan). Written informed consent from the families of all the autopsy subjects was obtained.

A total of 13 segments, obtained from the lesions responsible for mortality in the patients with AMI, were then separated into either ruptured $(n=7)$ or eroded $(n=6)$ plaques, according to the definition described previously (4). In the 13 patients with AMI, emergency PCI was performed in 7 patients. The interval between the AMI onset and mortality varied from 0 to 2 days (mean interval $<1$ day). No significant differences were observed between patients with ruptured or eroded plaques, with respect to age, sex, or risk factors.

The coronary arteries were dissected from the epicardial surface and a $2 \mathrm{~mm}$ slice from each segment was snap-frozen and stored at $-80^{\circ} \mathrm{C}$. The snap-frozen specimens were sectioned serially at $6 \mu \mathrm{m}$ thickness and fixed with acetone. Every first section was stained with hematoxylin-eosin; the other sections were used for immunohistochemical investigation.
Immunohistochemistry

Single staining. Table I presents the source, specificity and working dilution of the antibodies used. In the present study, monoclonal antibodies against human NPR-A and NPR-B were employed; the specificity of these antibodies has been reported previously (19). For the identification of NEP, anti-common acute lymphocytic leukemia antigen (CALLA; CD10) was used (20). The specificity of the results obtained with NPR-A, NPR-B or NEP was checked by omitting the primary antibodies and using non-immune mouse serum (DAKO; Agilent Technologies, Inc., Santa Clara, CA, USA) as a negative control. In the present immunohistochemical staining, a 3-step staining procedure was used, with the streptavidin-biotin complex method for color detection. Peroxidase activity was visualized by incubation with 3-amino-9-ethyl-carbazole for $10 \mathrm{~min}$ at room temperature, followed by faint counter-staining of the sections with hematoxylin.

Immunodouble staining. The simultaneous identification of SMCs and macrophages was performed on the basis of two primary antibodies of a different immunoglobulin $\mathrm{G}$ subclass (1A4 and CD68) (21). The enzymatic activity of $\beta$-galactosidase for 1A4 was visualized in turquoise (BioGenex kit, BioGenex, Fremont, CA, USA) while that of alkaline phosphatase for CD68 was visualized in red (New Fuchsin kit, DAKO; Agilent Technologies, Inc.). To identify cell types that express NPR-A or NPR-B, double immunostaining was performed between macrophages (CD68) and each of NPR-A and NPR-B. In addition, double immunostaining was also performed between neutrophils (CD66b) and each of NPR-A, NPR-B and NEP using modifications of procedures reported previously (21). In these double immunostainings, alkaline phosphatase was visualized with fast blue $\mathrm{BB}$ while peroxidase activity was identified by 3-amino-9-ethylcarbazole development.

Quantitative methods. The surface area containing NPR-A-positive cells and NPR-B-positive cells was quantified with the use of computer-aided planimetry (WinROOF2015, Mitani Corporation, Fukui, Japan) and the amount of NPR-Aor NPR-B-positive cells was estimated as a percentage of the total surface area of the tissue section. CD66b-positive neutrophil numbers and NEP-positive cell numbers were calculated in the entire tissue sections and expressed as the number of cells per $\mathrm{mm}^{2}$ of intimal tissue. NPR-A-, NPR-Bor NEP-positive cells, or neutrophils within thrombi or blood clots were excluded. In the morphometrical analysis, quantification and calculation were performed by a single investigator who was unaware of the histologic classification of the patients. The results are expressed as mean \pm standard deviation. Statistical comparison between 2 groups was performed using an unpaired Student's t-test or Mann-Whitney $\mathrm{U}$ test, as appropriate. $\chi^{2}$ test or Fisher's exact test was used for categorical variables. $\mathrm{P}<0.05$ was considered to indicate a statistically significant difference.

\section{Results}

\section{Immunocytochemistry}

Specimens obtained from patients with non-cardiovascular diseases. In the 20 normal coronary arteries with diffuse intimal thickening, macrophages were not detected. In the 12 early atherosclerotic lesions with hypercellularity, 5 lesions 


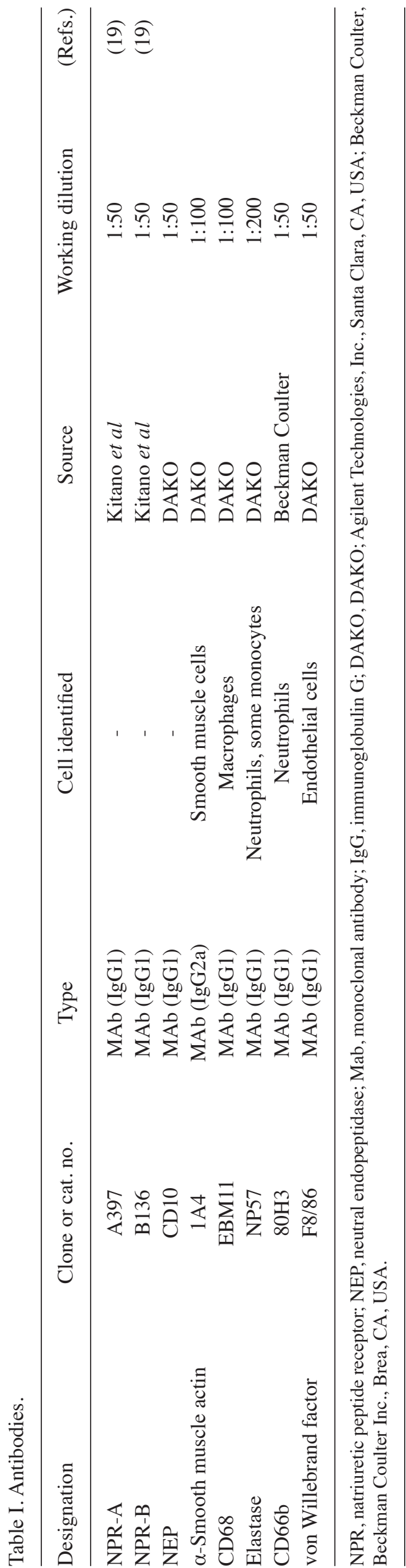

consisted predominantly of SMCs, while the other 7 lesions were characterized by the presence of foci of macrophages. In the 15 advanced fibrous plaques, scattered macrophages were identified in 5 lesions. In the 22 advanced fibrolipid plaques, abundant macrophages were observed in all lesions (Fig. 1A). Neutrophil infiltration was observed in 2 of the 22 advanced fibrolipid plaques. In the normal coronary arteries with diffuse intimal thickening, medial and intimal SMCs stained positive for NPR-A, but negative for NPR-B. A similar staining pattern was identified in medial SMCs of the early atherosclerotic lesions with hypercellularity. However, SMCs within these early atherosclerotic (hypercellular) lesions demonstrated distinct expression of NPR-A with occasional positive staining for NPR-B. In advanced fibrous and fibrolipid plaques, NPR-A expression was decreased markedly in intimal and medial SMCs and SMCs within the plaque demonstrated little or no expression of NPR-A (Fig. 1B). NPR-A positivity and NPR-B positivity were detected in accumulated macrophages within the plaques (Fig. 1B and C). In normal coronary arteries with diffuse intimal thickening, hypercellular lesions, or advanced fibrous plaques, NEP positivity was not observed. However, in the two advanced fibrolipid plaques with neutrophil infiltration, NEP positivity was identified in these neutrophils.

Specimens obtained from patients with AMI. Ruptured and eroded plaques contained numerous macrophages (Figs. 2A and B; 3A and B). Neutrophil infiltration was distinctly identified in all lesions with plaque rupture or erosion (Figs. 2C and $3 \mathrm{C}$ ). With respect to the number of neutrophils, no significant differences were observed between ruptured and eroded plaques. Regarding the neutrophil number in the culprit lesions, no significant differences were observed between patients with AMI with PCI and those without PCI. NPR-A and NPR-B were expressed in macrophages, in addition to in neutrophils in ruptured and eroded plaques (Figs. 2D and F; 3D and F). Double immunostaining for neutrophils and NPR-A or NPR-B demonstrated that the majority of NPR-A- or NPR-B-positive cells were neutrophils and NPR-A- or NPR-B-positivity was also identified in occasional macrophages (Figs. 2E and G; 3E and G). Regarding NEP expression, neutrophils were positive for NEP in ruptured plaques, while in eroded plaques the majority of the neutrophils were negative for NEP (Figs. $2 \mathrm{H}$ and $3 \mathrm{H}$ ). Morphometric analysis demonstrated that the percentage of NPR-A- and NPR-B- positive cells did not differ between ruptured and eroded plaques, while the number of NEP-positive cells in ruptured plaques was significantly higher compared with eroded plaques $(\mathrm{P}<0.0001$; Fig. 4). In ruptured and eroded plaques, NPR-A expression in intimal and medial SMCs was decreased.

\section{Discussion}

Plaque rupture or erosion has been demonstrated to be the most important mechanism that underlies the sudden onset of acute coronary syndromes. Several pathophysiological mechanisms may serve a significant role in the process of plaque disruption, including inflammation, rheological factors, circumferential wall stress and vasoconstriction.

The current study, based on frozen sections, is the first, to the best of the authors' knowledge, to demonstrate cells positive for NPR-A and NPR-B in ruptured and eroded plaques. A 
A

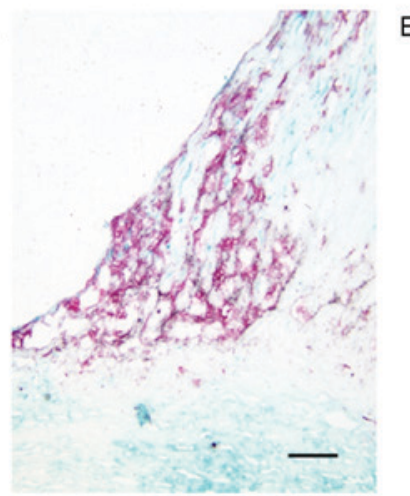

B

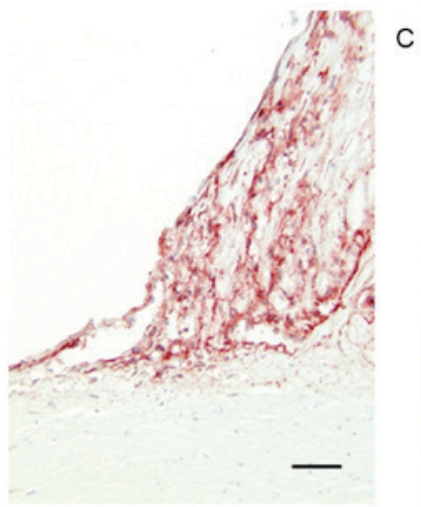

$C$

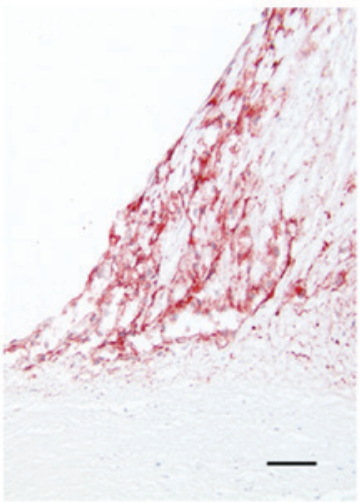

Figure 1. Micrographs showing an advanced atherosclerotic plaque. (A) Double immunostaining (SMC, turquoise; macrophage, red) reveals a lesion containing massive infiltration with macrophages. (B) The anti-NPR-A antibody indicated NPR-A expression in infiltrated macrophages, in contrast to only weak expression in intimal SMCs. (C) Anti-NPR-B antibody positivity indicating NPR-B expression in the infiltrated macrophages. Scale bars, $100 \mu \mathrm{m}$. SMC, smooth muscle cell; NPR, natriuretic peptide receptor.

A

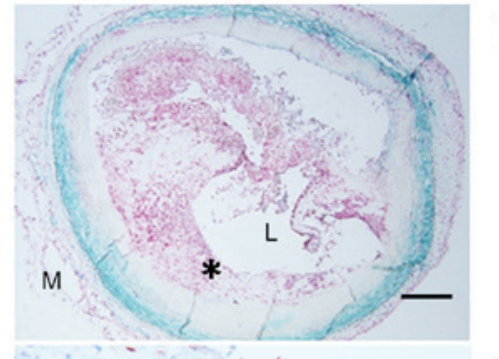

C

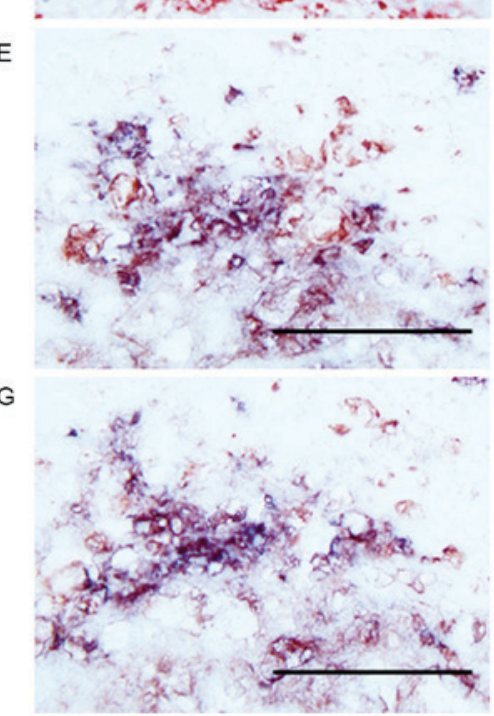

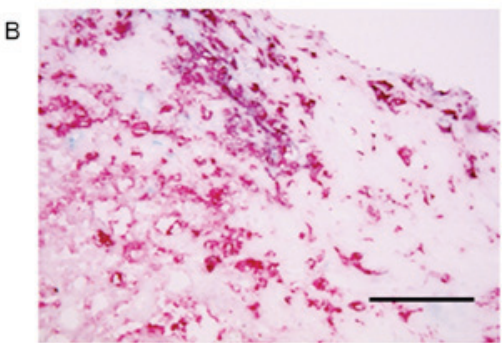

D

$\mathrm{F}$
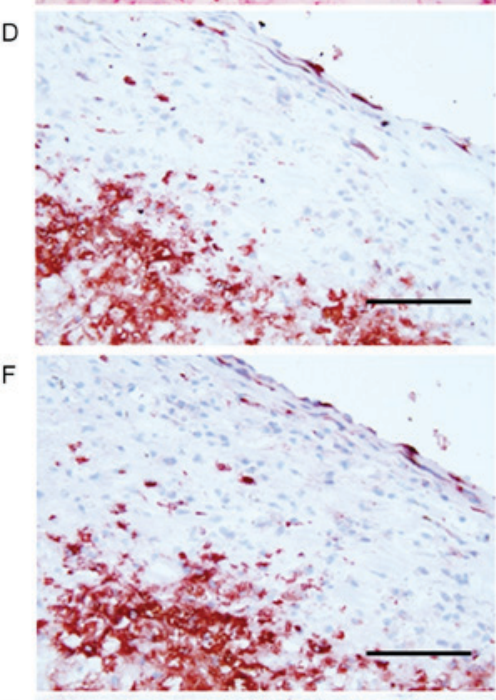

$\mathrm{H}$

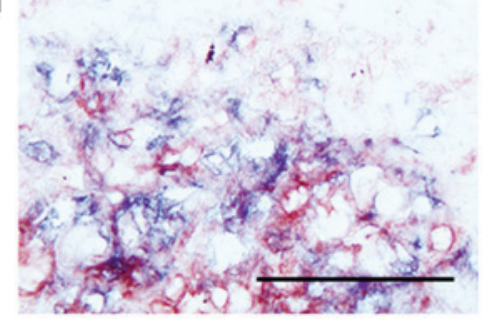

Figure 2. Micrographs of the site of plaque rupture obtained at autopsy in a patient with AMI. (A) Double immunostaining (SMC, turquoise; macrophage, red) showing a lipid-rich plaque containing numerous macrophages and a thin fibrous cap with SMCs. The area indicated by the asterisk is demonstrated at higher magnification in adjacent serial sections labeled B-H. (B) Double immunostaining (SMC, turquoise; macrophage, red) showing part of the lipid-rich plaque with numerous macrophages. (C) Anti-neutrophil CD66b antibody positivity in large numbers of neutrophils at the same site. (D) Anti-NPR-A antibody positivity indicating NPR-A expression in infiltrated macrophages and neutrophils. (E) Double immunostaining for neutrophils (blue) and NPR-A (red) showing the presence of NPR-A-positive neutrophils. (F) Anti-NPR-B antibody positivity indicating NPR-B expression in the majority of neutrophils in the plaque. (G) Double immunostaining for neutrophils (blue) and NRR-B (red) showing that almost all the cells are double stained (purple) and therefore are neutrophils (H) Double immunostaining (neutrophil CD66b, blue; NEP, red) indicated that almost all cells show double staining (purple), indicating that the NEP-positive cells are neutrophils. Scale bars: A, $500 \mu \mathrm{m}$; B-H, $100 \mu \mathrm{m}$. AMI, acute myocardial infarction; SMC, smooth muscle cell; NPR, natriuretic peptide receptor; L, lumen; M, media. 

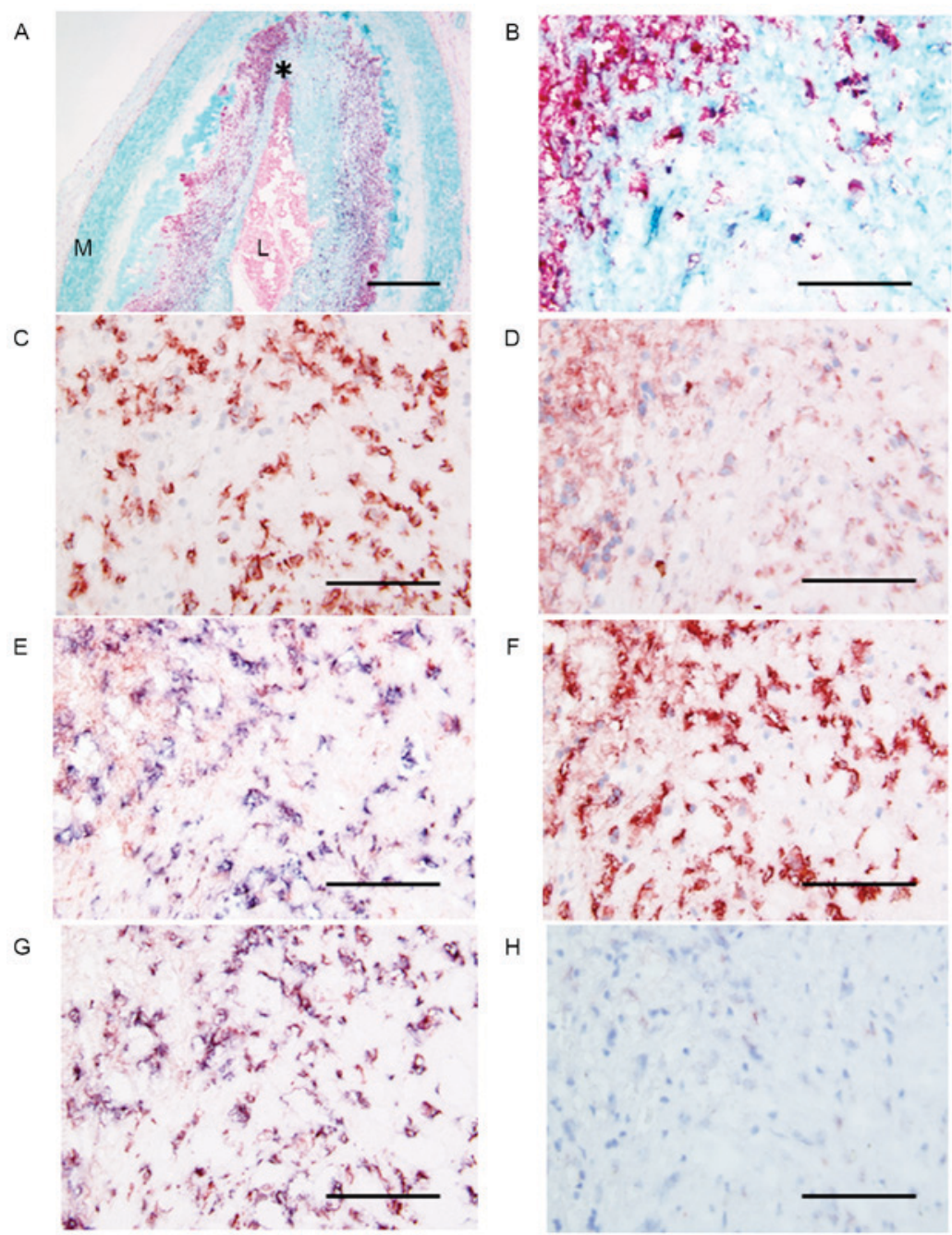

Figure 3. Micrographs of the site of plaque erosion obtained at autopsy in a patient with AMI. (A) Double immunostaining (SMC, turquoise; macrophage, red) indicates abundant macrophages within the plaque. $\mathrm{L}$, lumen, $\mathrm{M}$, media. The area indicated by the asterisk is demonstrated at a higher magnification in adjacent serial sections, labeled B-H. (B) Double immunostaining (SMC, turquoise; macrophage, red) indicates large numbers of macrophages. (C) Anti-neutrophil CD66b antibody positivity in large numbers of neutrophils at the same site. (D) Anti-NPR-A antibody positivity indicating NPR-A expression in infiltrated macrophages and neutrophils. (E) Double immunostaining for neutrophils (blue) and NPR-A (red) showing the presence of NPR-A-positive neutrophils (F) Anti-NPR-B antibody positivity indicating NPR-B expression in the majority of neutrophils in the plaque. (G) Double immunostaining for neutrophils (blue) and NRR-B (red) showing that almost all the cells are double stained (purple) and therefore are neutrophils. (H) Double immunostaining (neutrophil CD66b, blue; NEP, red) shows that all cells stain blue, indicating that these neutrophils are negative for NEP. Scale bars: A, $500 \mu \mathrm{m} ; \mathrm{B}-\mathrm{H}, 100 \mu \mathrm{m}$. AMI, acute myocardial infarction; SMC, smooth muscle cell; NPR, natriuretic peptide receptor.

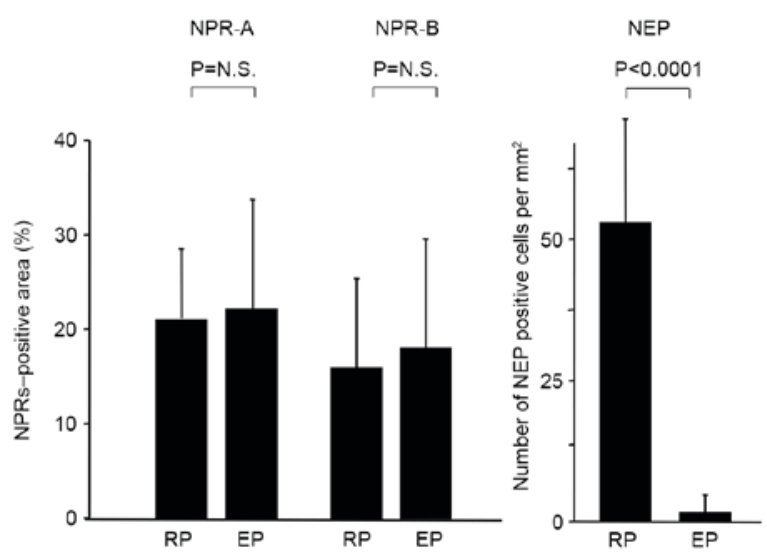

Figure 4. Graphs showing the NPR-A- and B-positive areas, expressed as a percentage of the total surface area and the number of NEP-positive cells, expressed as absolute numbers per $\mathrm{mm}^{2}$ of intimal tissue, in RPs $(\mathrm{n}=7)$ and EPs $(n=6)$. NPR, natriuretic peptide receptor; NEP, neutral endopeptidase; $\mathrm{RP}$, ruptured plaques; EP, eroded plaques. previous study (22) demonstrated the presence of mRNA and its translation products for NPs and their receptors in human coronary atherosclerotic plaques. However, the involvement of NPR-A and NPR-B in the development of the various types of coronary atherosclerotic lesions, including ruptured and eroded plaques, remains to be established. The findings of the present immunohistochemical study indicated that cell types involved in NPR-A and NPR-B expression levels in various stages of coronary atherosclerotic lesions are different. In early atherosclerotic lesions with hypercellularity, SMCs are predominantly involved, while in advanced atherosclerotic lesions NPR-A and NPR-B are mainly expressed in accumulated macrophages. In ruptured and eroded plaques the great majority of NPR-A- or NPR-B-positive cells are neutrophils. Previous experimental studies have demonstrated that NPR-A and NPR-B are expressed in macrophages (23), in bone marrow-derived stromal cells (24) and in neutrophils (13). These experimental data and the results of the present study 
suggest that NPR-A and NPR-B contribute to the progression of plaque instability in human coronary atherosclerotic lesions.

In the present study, no significant difference was observed in the expression of NPR-A and NPR-B in neutrophils between ruptured and eroded plaques. However, the number of NEP-positive neutrophils was markedly higher in ruptured plaques compared with eroded plaques, which is consistent with the results of our previous study (4). NEP can hydrolyze the NP family (6). NEP on the surface of leukocytes degrades the chemotactic peptide N-formyl-methionyl-leusyl-phenylaline and leukocyte adhesion, and chemotaxis is increased by inhibition of NEP $(6,20)$. Indeed, NEP-negative neutrophils exhibit a greater chemotactic reaction to the activated complement compared with NEP-positive neutrophils $(25,26)$. It has also been demonstrated that NEP expression occurs on fully mature neutrophils $(27,28)$. Perchansky et al (29) reported that NEP is detected only on fully mature and segmented neutrophils, while the majority of newly generated neutrophils exhibit a low expression of NEP due to the immaturity of the neutrophil membrane. Martens et al (30) further demonstrated that NEP expression in neutrophils was significantly decreased in patients with septic shock, which may be associated with an increase of immature neutrophils. These data are of interest, due to the fact that the results of the present study indicate that eroded plaques predominantly contain NEP-negative neutrophils. The biological significance of this phenomenon in human coronary atherosclerotic lesions remains to be elucidated. It may be hypothesized that the differences in NEP expression in neutrophils between ruptured and eroded plaques reflect differences in chemotaxis and, therefore, differences in the underlying inflammatory processes. In addition, the results of the present study indicated that the majority of the neutrophils in eroded plaques were negative for NEP, indicating that a rapid outburst of neutrophils had taken place, including that observed in the early stages of infection. In this context, it is conceivable that the underlying pathogenetic mechanism in plaque erosion is different from that in plaque rupture.

The roles of NPR-A and NPR-B expressed by neutrophils in ruptured and eroded plaques remain unclear. A previous study (13) demonstrated that neutrophils express NPR-A, the active receptor for NPs, and that ANP limits neutrophil activation via a cGMP-dependent mechanism. In addition, Mtairag et al (31) reported that ANP potentiation by NEP inhibition further limited neutrophil activation and neutrophil-vascular cell interactions. However, in those studies, the mechanism of the effect of ANP on neutrophils was not investigated in vivo. In the present study, strong expression of NPR-A and NPR-B was identified in neutrophils in ruptured and eroded plaques. However, ruptured plaques had a significantly higher number of NEP-positive neutrophils compared with eroded plaques. These observations suggested that the inhibitory effect of NPs on neutrophil activation can be suppressed by neutrophil NEP in ruptured plaques, while NPs can limit neutrophil activation via NPR-A and NPR-B more markedly in eroded plaques. Therefore, it can be hypothesized that the enhanced expression of NPR-A and NPR-B on NEP-negative neutrophils in eroded plaques may regulate inflammatory process and vascular activity in these lesions.

Atherosclerosis is a complex phenomenon associated with interaction of numerous factors. The NP system and its receptors are not the only factors involved, therefore, other factors must be taken into consideration to determine the functional significance of this system. Nevertheless, the present study provided data to implicate NPR-A and NPR-B in the changes in vasomotor activity and inflammatory cell infiltration that occur in plaque instability.

In conclusion, distinct expression of NPR-A and NPR-B in culprit lesions underlying AMI strongly suggests that NPs serve a role in regulating plaque instability in humans.

\section{References}

1. Davies MJ and Thomas AC: Plaque fissuring-the cause of acute myocardial infarction, sudden ischemic mortality, and crescendo angina. Br Heart J 53: 363-373, 1985.

2. Fuster V, Badimon L, Badimon JJ and Chesebro JH: The pathogenesis of coronary artery disease and the acute coronary syndromes. N Engl J Med 326: 242-250, 1992.

3. van der Wal AC, Becker AE, van der Loos CM and Das PK: Site of intimal rupture or erosion of thrombosed coronary atherosclerotic plaques is characterized by an inflammatory process irrespective of the dominant plaque morphology. Circulation 89: 36-44, 1994.

4. Naruko T, Ueda M, Haze K, van der Wal AC, van der Loos CM, Itoh A, Komatsu R, Ikura Y, Ogami M, Shimada Y, et al: Neutrophil infiltration of culprit lesions in acute coronary syndromes. Circulation 106: 2894-2900, 2002.

5. Kayo S, Ohsawa M, Ehara S, Naruko T, Ikura Y, Hai E, Yoshimi N, Shirai N, Tsukamoto Y, Itabe H, et al: Oxidized low-density lipoprotein levels circulating in plasma and deposited in the tissues: Comparison between Helicobacter pylori-associated gastritis and acute myocardial infarction. Am Heart J 148: 818-825, 2004.

6. Connelly JC, Skidgel RA, Schulz WW, Johnson AR and Erdos EG: Neutral endopeptidase 24.11 in human neutrophils: Cleavage of chemotactic peptide. Proc Natl Acad Sci USA 82: 8737-8741, 1985.

7. Galvani M, Ferrini D and Ottani F: Natriuretic peptides for risk stratification of patients with acute coronary syndromes. Eur J Heart Fail 6: 327-333, 2004.

8. Suzuki S, Yoshimura M, Nakayama M, Mizuno Y, Harada E, Ito T, Nakamura S, Abe K, Yamamuro M, Sakamoto T, et al: Plasma level of B-type natriuretic peptide as a prognostic marker after acute myocardial infarction: A long-term follow-up analysis. Circulation 110: 1387-1391, 2004.

9. Chang MS, Lowe DG, Lewis M, Hellmiss R, Chen E and Goeddel DV: Differential activation by atrial and brain natriuretic peptides of two different receptor guanylate cyclases. Nature 341: 68-72, 1989.

10. MaackT,Suzuki M,AlmeidaFA, Nussenzveig D,Scarborough RM, McEnroe GA and Lewicki JA: Physiological role of silent receptors of atrial natriuretic factor. Science 238: 675-678, 1987.

11. Naruko T, Ueda M, van der Wal AC, van der Loos CM, Itoh H, Nakao K and Becker AE: C-type natriuretic peptide in human coronary atherosclerotic lesions. Circulation 94: 3103-3108, 1996.

12. Naruko T, Itoh A, Haze K, Ehara S, Fukushima H, Sugama Y, Shirai N, Ikura Y, Ohsawa M and Ueda M: C-Type natriuretic peptide and natriuretic peptide receptors are expressed by smooth muscle cells in the neointima after percutaneous coronary intervention. Atherosclerosis 181: 241-250, 2005.

13. Matsumura T, Kugiyama K, Sugiyama S, Ohgushi M, Amanaka K, Suzuki M and Yasue H: Neutral endopeptidase 24.11 in neutrophils modulates protective effects of natriuretic peptides against neutrophils-induced endothelial cytotoxity. J Clin Invest 97: 2192-2203, 1996

14. Wiedermann CJ, Niedermühlbichler M, Braunsteiner $\mathrm{H}$ and Widermann CJ: Priming of polymorphonuclear neutrophils by atrial natriuretic peptide in vitro. J Clin Invest 89: 1580-1586, 1992.

15. Garlichs CD, Zhang H, Schmeisser A and Daniel WG: Priming of superoxide anion in polymorphonuclear neutrophils by brain natriuretic peptide. Life Sci 65: 1027-1033, 1999.

16. Izumi T, Saito Y, Kishimoto I, Harada M, Kuwahara K, Hamanaka I, Takahashi N, Kawakami R, Li Y, Takemura G, et al: Blockade of the natriuretic peptide receptor guanylyl cyclase-A inhibits NF-kappaB activation and alleviates myocardial ischemia/reperfusion injury. J Clin Invest 108: 203-213, 2001. 
17. Stary HC, Chandler AB, Dinsmore RE, Fuster V, Glagov S, Insull W Jr, Rosenfeld ME, Schwartz CJ, Wagner WD and Wissler RW: A definition of advanced types of atherosclerotic lesions and a histological classification of atherosclerosis. A report from the Committee on Vascular Lesions of the Council on Arteriosclerosis, American Heart Association. Arterioscler Thromb Vasc Biol 15: 1512-1531, 1995.

18. Stary HC, Chandler AB, Glagov S, Guyton JR, Insull W Jr Rosenfeld ME, Schaffer SA, Schwartz CJ, Wagner WD and Wissler RW: A definition of initial, fatty streak, and intermediate lesions of atherosclerosis. A report from the committee on vascular lesions of the council on arteriosclerosis, American Heart Association. Arterioscler Thromb 14: 840-856, 1994.

19. Kitano K, Fukuda Y, Nagahira K, Nasu T, Izumi R, Kawashima K and Nakanishi T: Production and characterization of monoclonal antibodies against human natriuretic peptide receptor-A or -B. Immunol Lett 47: 215-222, 1995.

20. Letarte M, Vera S, Tran R, Addis JB, Onizuka RJ, Quackenbush EJ, Jongeneel CV and McInnes RR: Common acute lymphocytic leukemia antigen is identical to neutral endopeptidase. J Exp Med. 168: 1247-1253, 1988

21. van der Loos CM, Becker AE and van den Oord JJ: Practical suggestions for successful immunoenzyme double-staining experiments. Histochem J 25: 1-13, 1993.

22. Casco VH, Veinot JP, Kuroski de Bold ML, Masters RG, Stevenson MM and de Bold AJ: Natriuretic peptide system gene expression in human coronary arteries. J Histochem Cytochem 50: 799-809, 2002

23. Kiemer AK and Vollmar AM: Effects of different natriuretic peptides on nitric oxide synthesis in macrophages. Endocrinology 138: 4282-4290, 1997.

24. Agui T, Yamada T, Legros G, Nakajima T, Clark M, Peschel C and Matsumoto $\mathrm{K}$ : Expression of receptors for atrial natriuretic peptide on the murine bone marrow-derived stromal cells. Endocrinology 130: 2487-2494, 1992.

25. McCormack RT, Nelson RD, Chenoweth DE and LeBien TW: Identification and characterization of a unique subpopulation (CALLA/CD10-negative) of human neutrophils manifesting a heightened chemotactic response to activated complement. Blood 70: 1624-1629, 1987.
26. Braun MP, Martin PJ, Ledbetter JA and Hansen JA: Granulocytes and cultured human fibroblasts express common acute lymphoblastic leukemia-associated antigen. Blood 61: 718-725, 1983

27. Tran-Paterson R, Boileau $G$, Giguère $V$ and Letarte $M$ Comparative levels of CALLA/neutral endopeptidase on normal granulocytes, leukemic cells, and transfected COS-1 cells. Blood 76: 775-782, 1990.

28. McCormack RT, Nelson RD, Solem LD and LeBien TW: Decreased expression of the common acute lymphoblastic leukemia antigen (CALLA/CD10) on neutrophils from patients with thermal injury. Br J Haematol 69: 189-195, 1988.

29. Perchansky L, Pirrotta V and Kaplan S: Flow cytometric study of the expression of neutral endopeptidae (CD10/CALLA) on the surface of newborn granulocytes. Modern Pathol 6: 414-418, 1993.

30. Martens A, Eppink GJ, Woittiez AJ, Eidhof H and de Leij LF: Neutrophil function capacity to express CD10 is decreased in patients with septic shock. Crit Care Med 27: 549-553, 1999.

31. Mtairag el M, Houard X, Rais S, Pasquier C, Oudghiri M, Jacob MP, Meilhac O and Michel JB: Pharmacological potentiation of natriuretic peptide limits polymorphonuclear neutrophil-vascular cell interactions. Arterioscler Thromb Vasc Biol 22: 1824-1831, 2002. 